\title{
Radiopharmaceutical Medicinal Product
}

National Cancer Institute

\section{Source}

National Cancer Institute. Radiopharmaceutical Medicinal Product. NCI Thesaurus. Code C142659.

A radioactive compound that is administered to patients for diagnostic or therapeutic purposes. 